\title{
Case Report: Subarachnoid Hemorrhage and Eosinophilic Meningitis due to Disseminated Fascioliasis
}

\author{
Gareth Hughes, ${ }^{1 \star}$ Chris A. Green, ${ }^{1,2}$ Duncan Street, ${ }^{3}$ Yasmine Maurice, ${ }^{4}$ John Henderson, ${ }^{5}$ Andrew Woodhouse, ${ }^{1}$ David Nicholl, ${ }^{3}$ \\ and James E. Scriven ${ }^{1}$ \\ ${ }^{1}$ Department of Infectious Diseases and Tropical Medicine, University Hospitals Birmingham NHS (National Health Service) Trust, Birmingham \\ Heartlands Hospital, Birmingham, United Kingdom; ${ }^{2}$ Institute of Microbiology and Infection, University of Birmingham, Birmingham, United \\ Kingdom; ${ }^{3}$ Department of Neurology, Sandwell General Hospital, West Bromwich, United Kingdom; ${ }^{4}$ Department of Cellular Pathology, University \\ Hospitals Birmingham NHS Trust, Birmingham Heartlands Hospital, Birmingham, United Kingdom; ${ }^{5}$ Department of Radiology, University Hospitals \\ Birmingham NHS Trust, Birmingham Heartlands Hospital, Birmingham, United Kingdom
}

\begin{abstract}
Human infection with the trematode Fasciola occurs with a worldwide prevalence of up to $17 \mathrm{million}$. Sheep and cattle are the normal host. Infection typically results in hepatobiliary disease, but extrahepatic manifestations are occasionally reported. Here, we present the case of a previously healthy 31-year-old Kurdish woman, admitted to hospital with a subarachnoid hemorrhage, eosinophilic meningitis, and lung and liver disease. A diagnosis of Fasciola infection was made based on strongly positive serology in blood and cerebrospinal fluid. The patient improved following treatment with triclabendazole and prednisolone.
\end{abstract}

\section{INTRODUCTION}

Eosinophilic meningitis is defined as a cerebrospinal fluid (CSF) eosinophil count of $>10 / \mathrm{mm}^{3}$ and/or $>10 \%$ of CSF leukocytes. ${ }^{1}$ It is most commonly associated with invasive helminthic infections caused by Angiostrongylus cantonensis, Gnathostoma spinigerum, and Baylisascaris procyonis but has also been occasionally reported in fungal and bacterial infections caused by Coccidioides immitis, Treponema pallidum, and Mycobacterium tuberculosis. ${ }^{1}$ Noninfective causes include central nervous system lymphoma, multiple sclerosis, and reaction to foreign material. ${ }^{2}$ Here, we report a case of disseminated Fasciola infection presenting with subarachnoid hemorrhage and eosinophilic meningitis with evidence of liver and lung pathology.

\section{CASE REPORT}

A previously healthy 31-year-old woman presented to hospital with a 2-day history of right frontal headache, abdominal pain, vomiting, and fever. She was from the Darbandikhan region of northern Iraq and had migrated to the UK 4 months previously. Physical examination revealed a temperature of $37.9^{\circ} \mathrm{C}$, right upper quadrant and epigastric tenderness, and mild bilateral papilledema without any focal neurological deficit. Her routine blood tests showed a modest neutrophilia $\left(11.4 \times 10^{9} / \mathrm{L}\right)$, mild eosinophilia $\left(1.69 \times 10^{9} / \mathrm{L}\right)$, raised alkaline phosphatase (176 IU/L), and mild hyperbilirubinemia (46 $\mu \mathrm{mol} / \mathrm{L})$ but were otherwise unremarkable. computerized tomography $(\mathrm{CT})$ and subsequent magnetic resonance $(\mathrm{MRI})$ imaging of her brain identified an acute right frontal subarachnoid hemorrhage with a $2.3 \times 1.7 \times 1.4-\mathrm{cm}$, nonenhancing arachnoid cyst in the posterior cranial fossa causing anterior displacement of the cerebellum (concluded to be an anatomical variant). Small ischemic infarcts were seen in the right middle cerebral artery territory, but there was no evidence of aneurysmal disease (Figure 1). Lumbar

*Address correspondence to Gareth Hughes, Department of Infectious Diseases and Tropical Medicine, University Hospitals Birmingham NHS Trust, Birmingham Heartlands Hospital, Birmingham B9 5SS, United Kingdom. E-mail: g.hughes3@nhs.net puncture (LP) and CSF analysis confirmed the presence of xanthochromia along with a pleocytosis of $326 \mathrm{cells} / \mathrm{mm}^{3}(70 \%$ polymorphs and $30 \%$ mononuclear), elevated protein $(1.63 \mathrm{~g} / \mathrm{L})$ and low glucose $(1.5 \mathrm{mmol} / \mathrm{L}$, compared with blood glucose $6.6 \mathrm{mmol} / \mathrm{L}$ ) (Table 1). Cerebrospinal fluid Gram stain, CSF bacterial culture, and peripheral blood culture were all negative.

A presumptive diagnosis of bacterial meningitis was made and she was treated with intravenous ceftriaxone $2 \mathrm{~g}$ twice daily, but her symptoms persisted, and after 7 days, she was changed to intravenous meropenem $2 \mathrm{~g}$ three times daily. Repeat LP 9 days after presentation showed a reduction in CSF cell count and protein, but CSF glucose remained low (Table 1). Polymerase chain reaction testing of CSF was negative for herpes simplex virus (HSV)-1/2, varicella zoster virus (VZV), enterovirus, Streptococcus pneumoniae, Neisseria meningitidis, and M. tuberculosis (GeneXpert $^{\circledR}$, Cepheid, Sunnyvale, CA). Cerebrospinal fluid was also negative for cryptococcal antigen and Borrelia burgdorferi antibodies (immunoblot lgG). Peripheral blood serology was negative for HIV-1/2, T. pallidum, and B. burgdorferi. Computerized tomography imaging of chest and abdomen revealed a hypodense liver lesion (Figure 1D) along with consolidation and cavitation in the left lower lobe of the lung (Figure 1E). Bronchoscopy and bronchoalveolar lavage were performed and samples sent for bacterial and mycobacterial culture, and no significant pathogens were identified. Cytology of her bronchoalveolar lavage revealed numerous macrophages, metaplastic cells, and respiratory epithelial cells.

She completed a total of 4 weeks of intravenous antibiotics but did not improve. A further LP was performed that identified a rising CSF white cell count and rising protein level (Table 1). Additional CSF was sent for mycobacterial culture, and she was started on empirical four-drug antituberculous therapy (rifampicin/isoniazid/pyrazinamide/ethambutol), pyridoxine, and prednisolone $60 \mathrm{mg}$ daily. Subsequently, an ultrasound-guided biopsy of the hepatic lesion was performed, which revealed an eosinophilic infiltrate within the vessels of the portal tract (Figure 2). A further LP was performed for CSF cytology which identified numerous eosinophilic granulocytes $(80 \%$ of the whole cell population), activated lymphocytes, and plasma cells (Figure 2). No malignant cells were observed. 

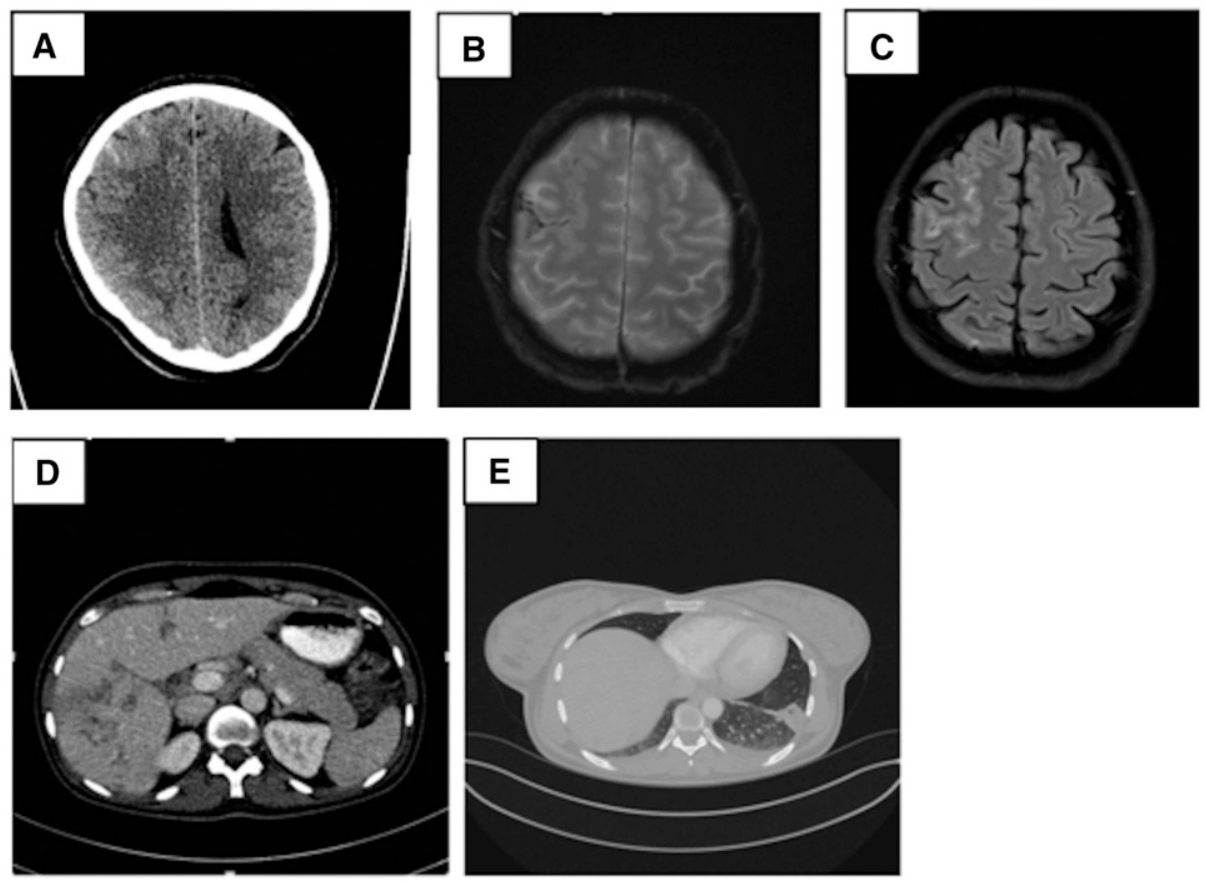

FIGURE 1. (A) CT scan showing a new subarachnoid hemorrhage in the right anterior parietal lobe. (B) MRI T2 gradient echo showing susceptibility artifact in keeping with the subarachnoid hemorrhage. (C) MRI fluid attenuated inversion recovery (FLAIR) sequence showing bright sulci with features in keeping with the subarachnoid hemorrhage. (D) CT scan showing ill-defined low-attenuation lesions in both hepatic lobes, suggestive of inflammatory foci. (E) CT scan showing the focal area of consolidation and possible liquefaction of the left lower lobe of the lung.

Subsequent serology testing revealed high titers of Fasciolaspecific IgG antibody (DRG diagnostics ${ }^{\circledR}$, Marburg, Germany) in the serum and CSF but negative antibodies to Toxocara, Echinococcus, Amoeba, Schistosoma, and Strongyloides. Microscopic examination of stool samples did not detect any parasitic ova.

Antituberculous treatment was stopped but steroids were continued, and she was treated with triclabendazole $10 \mathrm{mg} / \mathrm{kg}$ daily for 3 days with good effect. Her headaches improved, and she was discharged on a tapering course of steroids over 4 weeks. After peaking at $1.74 \times 10^{9} / \mathrm{L}$, the peripheral eosinophilia decreased to $0.02 \times 10^{9} / \mathrm{L}$, 2 weeks after treatment with triclabendazole.

Subsequent questioning of her food intake before her move to the United Kingdom identified regular consumption of watercress from a local river near her family home in Iraq.

\section{DISCUSSION}

Fascioliasis, caused by infection with the tremotodes Fasciola hepatica or Fasciola gigantica, has an estimated global prevalence of 2.4-17 million. The distribution is worldwide, but the most commonly affected countries include Bolivia, Peru, Egypt, France, Iran, and Portugal. ${ }^{3}$ In more recent years, there have been reports of cases in northeast Iraq. ${ }^{4}$ Ruminants such as sheep, goats, or cattle are the definitive host, but humans can be accidentally infected following the ingestion of metacercariae from uncooked freshwater vegetation, crabmeat, and khat. ${ }^{5-7}$ Metacercariae migrate from the bowel via the peritoneal cavity to the liver and bilary tract, where they reach adulthood and can measure $3 \mathrm{~cm} \times 1.5 \mathrm{~cm}$. Disease occurs because of focal inflammation and hemorrhage caused by the direct migration of metacercariae through the bowel, peritoneum, and liver, and the persistent presence of the adult worm in the biliary system (where it can survive up to 10 years), resulting in chronic cholangitis and fibrosis. ${ }^{5}$ Clinical features of the infection include abdominal pain, fever, gastrointestinal upset, hepatomegaly, urticaria, and peripheral eosinophilia. Computerized tomography or ultrasound scanning may identify hypodense areas in the liver tissue and occasionally evidence of a dilated/obstructed biliary system and/or

TABLE 1

Serial measurements of cerebrospinal fluid during inpatient stay

\begin{tabular}{|c|c|c|c|c|}
\hline Date & February 13, 2018 & February 22, 2018 & March 15, 2018 & March 21, 2018 \\
\hline Opening pressure (range: $7-18 \mathrm{~cm} \mathrm{H}_{2} \mathrm{O}$ ) & Not performed & $20.5 \mathrm{~cm} \mathrm{H}_{2} \mathrm{O}$ & $21 \mathrm{~cm} \mathrm{H}_{2} \mathrm{O}$ & $26 \mathrm{~cm} \mathrm{H}_{2} \mathrm{O}$ \\
\hline CSF appearance & Clear and colorless & Straw colored & Turbid & Turbid \\
\hline $\begin{array}{l}\text { CSF/blood glucose (range: } 4-11 \mathrm{mmol} / \mathrm{L} \\
\text { for blood with CSF within } 1 \mathrm{mmol} / \mathrm{L} \text { of } \\
\text { this value) }\end{array}$ & $1.5 / 6.6 \mathrm{mmol} / \mathrm{L}$ & $1.4 / 3.3 \mathrm{mmol} / \mathrm{L}$ & $0.4 / 4.8 \mathrm{mmol} / \mathrm{L}$ & $0.5 / 5.7 \mathrm{mmol} / \mathrm{L}$ \\
\hline CSF protein (range: $0.1-0.4 \mathrm{~g} / \mathrm{L}$ ) & $1.63 \mathrm{~g} / \mathrm{L}$ & $0.69 \mathrm{~g} / \mathrm{L}$ & $1.94 \mathrm{~g} / \mathrm{L}$ & $2.60 \mathrm{~g} / \mathrm{L}$ \\
\hline CSF cells & & & & \\
\hline White cells (range: $0-5$ cells $/ \mathrm{mm}^{3}$ ) & 326 & 180 & 520 & 800 \\
\hline Polymorphs (\%) & 70 & - & 10 & 55 \\
\hline Lymphocytes (\%) & 30 & 100 & 90 & 45 \\
\hline
\end{tabular}



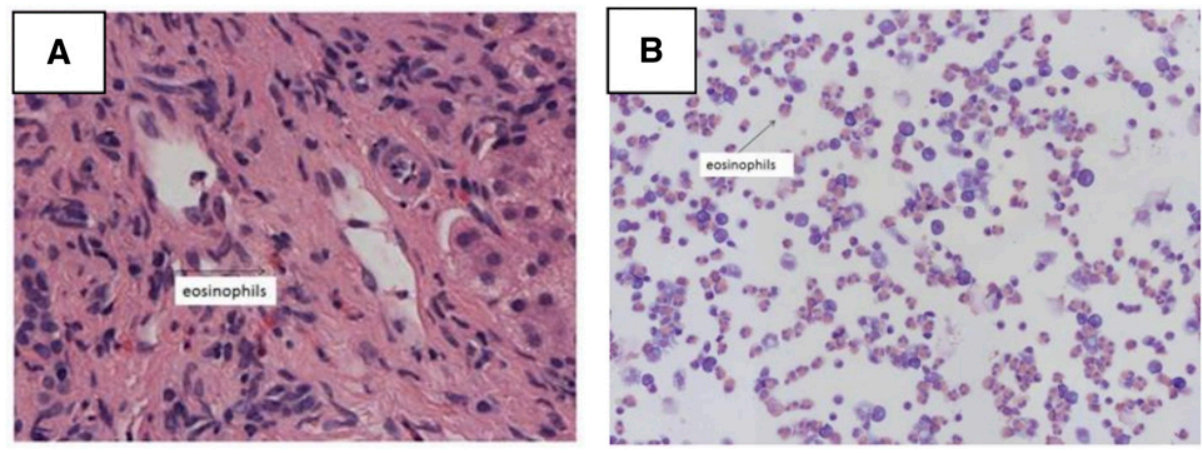

FIGURE 2. (A) Liver biopsy ( $\times 40$; hematoxylin and eosin [H\&E] stain) showing eosinophilic infiltrates. (B) Cerebrospinal fluid ( $\times 10$; May-Grünwald Giemsa [MGG] stain) showing $80 \%$ eosinophilia. This figure appears in color at www.ajtmh.org.

ascites. ${ }^{4,5}$ Erroneous migration of larvae can rarely lead to extrahepatic manifestations in a variety of organs, including the heart, lungs, subcutaneous tissue, pancreas, spleen, eyes, and brain. Symptoms of neurological infection include headache and a variety of focal neurological symptoms. In some untreated cases, this can persist for months or years and may lead to death or significant neurological sequelae. ${ }^{3}$ Cerebral infarction, subdural hemorrhage, and intracerebral hemorrhage have been reported, but isolated subarachnoid hemorrhage is rare. ${ }^{3}$

Erroneous migration of ova to the brain parenchyma is recognized with other trematode infections such as schistosomiasis and has been reported to occur in up to $26 \%$ of patients in a postmortem study. ${ }^{8}$ A proposed mechanism for this is the direct spread of infection via the valveless network of veins connecting the Batson venous plexus (deep pelvic and thoracic veins) to vertebral venous plexuses. ${ }^{8}$ However, in fascioliasis, it is the larvae and mature adults that migrate, resulting in direct tissue damage.

The diagnosis of fascioliasis is usually made by identification of ova or adult flukes in stool or sterile tissue specimens. However, sensitivity is low and can be falsely negative in the acute stage and in ectopic infection. ${ }^{9}$ Stool examination was negative in our patient on two occasions, and the diagnosis was based on the findings of eosinophilic disease in the liver and central nervous system, radiological evidence of lung involvement, a high titer for anti-Fasciola IgG antibodies in the serum and CSF, and a history of migration from an endemic region. Serology testing for helminth infections can be complicated by cross-reactivity, particularly within species of the same class such as the Trematoda causing infections such as clonorchiasis, paragonimiasis, and schistosomiasis. ${ }^{10,11}$ However, validation studies of this specific assay have reported a sensitivity and specificity of $95.3 \%(95 \% \mathrm{Cl}$ 82.9-99.2) and $95.7 \%$ (95\% Cl 92.3-97.5), respectively. ${ }^{9}$ Occasional cross-reactions are reported with sera from patients with schistosomiasis, echinococcosis, cysticercosis, trichinosis, strongyloidiasis, and histoplasmosis, but none of these infections fitted with the clinical picture.

Although there are occasional reports of spontaneous resolution of neurofascioliasis, antihelminthic treatment with triclabendazole is recommended; nitazoxanide is an alternative. ${ }^{12}$ In our patient, we combined triclabendazole with corticosteroid therapy because of concerns about a potential paradoxical inflammatory reaction following the initiation of antihelminthic therapy. Paradoxical deterioration after initiation of antihelminthic therapy is a recognized complication in neurocysticercosis, and both the Infectious Diseases
Society of America and the American Society of Tropical Medicine and Hygiene recommend the use of corticosteroids alongside antihelminthic therapy to reduce the likelihood of seizures. ${ }^{13-15}$ Corticosteroids are also beneficial in the treatment of Angiostrongylus infection, where clinical trials have shown that their use results in significantly faster resolution of neurological symptoms. ${ }^{16}$ No trials of corticosteroids have been performed in neurofascioliasis, but there are anecdotal reports of an apparently beneficial effect. ${ }^{3}$

This case illustrates an unusual neurological complication of fascioliasis and highlights the potential importance of cytological examination of the CSF to correctly determine the nature of a CSF polymorphonuclear leukocyte infiltrate. It also demonstrates the benefits of serological testing to aid diagnosis. Further work is required to understand whether corticosteroids have a beneficial role as an adjunct to antihelminthic therapy in neurofascioliasis.

Received May 9, 2019. Accepted for publication July 29, 2019.

Published online January 13, 2020.

Acknowledgments: We would like to thank the patient for her consent to publish this case and also Peter Chiodini and Tim O'Dempsy for their additional advice in her management.

Authors' addresses: Gareth Hughes, Andrew Woodhouse, and James E. Scriven, Department of Infectious Diseases and Tropical Medicine, University Hospitals Birmingham NHS Trust, Birmingham Heartlands Hospital, Birmingham, United Kingdom, E-mails: g.hughes3@nhs.net, andrew.woodhouse@heartofengland.nhs.uk, and james.scriven@ heartofengland.nhs.uk. Chris A. Green, Department of Infectious Diseases and Tropical Medicine, University Hospitals Birmingham NHS Trust, Birmingham Heartlands Hospital, Birmingham, United Kingdom, and Institute of Microbiology and Infection, University of Birmingham, Birmingham, United Kingdom, E-mail: cgreen16@ nhs.net. Duncan Street and David Nicholl, Department of Neurology, Sandwell General Hospital, West Bromwich, United Kingdom, E-mails: duncan.street@nhs.net and david.nicholl@nhs.net. Yasmine Maurice, Department of Cellular Pathology, University Hospitals Birmingham NHS Trust, Birmingham Heartlands Hospital, Birmingham, United Kingdom, E-mail: yasmine.maurice@heartofengland.nhs.uk. John Henderson, Department of Radiology, University Hospitals Birmingham NHS Trust, Birmingham Heartlands Hospital, Birmingham, United Kingdom, E-mail: john.henderson@heartofengland.nhs.uk.

\section{REFERENCES}

1. Lo Re V 3rd, Gluckman SJ, 2003. Eosinophilic meningitis. Am J Med 114: 217-223.

2. Ramirez-Avila L, Slome S, Schuster FL, Gavali S, Schantz PM, Sejvar J, Glaser CA, 2009. Eosinophilic meningitis due to Angiostrongylus and Gnathostoma species. Clin Infect Dis 48: 322-327. 
3. Mas-Coma S, Agramunt VH, Valero MA, 2013. Direct and indirect affection of the central nervous system by Fasciola infection. Handb Clin Neurol 114: 297-310.

4. Hassan HA, Majid RA, Rashid NG, Nuradeen BE, Abdulkarim QH, Hawramy TA, Rashid RM, Farris AB, Guarner J, Hughson MD, 2013. Eosinophilic granulomatous gastrointestinal and hepatic abscesses attributable to basidiobolomycosis and fasciolias: a simultaneous emergence in Iraqi Kurdistan. BMC Infect Dis 13: 91.

5. Mandell GL, Bennett JE, Dolin R, Douglas RG, 2015. Trematodes. Mandell, Douglas and Bennett's Principles and Practice of Infectious Diseases, 8th edition. Philadelphia, PA: Elsevier, 3216-3226.

6. Zhou L, Luo L, You C, Wang B, Xu J, Liao L, Hui X, Cai B, 2008. Multiple brain hemorrhages and hematomas associated with ectopic fascioliasis in brain and eye. Surg Neurol 69: 516-521.

7. Green EW, Partridge DG, Green ST, McKendrick MW, 2009. Increasing prevalence of human fascioliasis (and an association with khat usage) in Sheffield, UK: a clinical and epidemiological case series. J Infect 59: S449.

8. Pittella JE, Lana-Peixoto MA, 1981. Brain involvement in hepatosplenic Schistosomiasis mansoni. Brain 104: 621-632.

9. Adela Valero M, Victoria Periago M, Perez-Crespo I, Rodríguez E, Perteguer MJ, Gárate T, González-Barberá EM, Mas-Coma S, 2012. Assessing the validity of an ELISA test for the serological diagnosis of human fascioliasis in different epidemiological situations. Trop Med Int Health 17: 630-636.
10. Jin Y, Kim EM, Choi MH, Oh MD, Hong ST, 2017. Significance of serology by multi-antigen ELISA for tissue helminthiases in Korea. J Korean Med Sci 32: 1118-1123.

11. Kinkel HF, Dittrich S, Baümer B, Weitzel T, 2012. Evaluation of eight serological tests for diagnosis of imported schistosomiasis. Clin Vaccine Immunol 19: 948-953.

12. WHO, 2007. Report of the WHO Informal Meeting on Use of Triclabendazole in Fascioliasis Control. Available at: http:// www.who.int/foodborne_trematode_infections/fascioliasis/ en/. Accessed February 26, 2019.

13. Garcia HH, Pretell MD, Gilman RH, Martinez SM, Moulton LH, Del Brutto OH, Herrera G, Evans CA, Gonzalez AE; Cysticercosis Working Group in Peru, 2004. A trial of antiparasitic treatment to reduce the rate of seizures due to cerebral cysticercosis. N Engl J Med 350: 249-258.

14. Garcia HH, Nash TE, Del Brutto $\mathrm{OH}, 2014$. Clinical symptoms, diagnosis and treatment of neurocysticercosis. Lancet Neurol 13: $1202-1215$.

15. White AC, Coyle CM, Rajshekhar V, Singh G, Hauser WA, Mohanty A, Garcia HH, Nash TE, 2018. Diagnosis and treatment of neurocysticercosis: 2017 clinical practice guidelines by the Infectious Diseases Society of America (IDSA) and the American Society of Tropical Medicine and Hygiene (ASTMH). Clin Infect Dis 66: e49-e75.

16. Chotmongkol V, Sawanyawisuth K, Thavornpitak Y, 2000. Corticosteroid treatment of eosinophilic meningitis. Clin Infect Dis 31: 660-662. 